\title{
New Vertical Geodesy
}

\author{
JAMES H. WhITCOMB \\ Seismological Laboratory, California Institute of Technology, Pasadena, California 91125
}

\begin{abstract}
Vertical geodesy is undergoing a revolution because of two factors. First, new precise three-dimensional position measurement techniques used over very long distances and based on extraterrestrial reference systems provide a new class and precision of geometric data previously unavailable for geophysical investigations. Second, physical models in tectonic theory for large earthquakes predict crustal distortions that violate the conventional assumptions used to interpret gravity and leveling data. Leveling and geometric elevation measurements are not directly comparable because the interpretation of leveling data is density-model dependent. Estimates of pre-1971 San Fernando earthquake elevation changes based on leveling of about $10 \mathrm{~cm}$ may be as much as $3 \mathrm{~cm}$, or $40 \%$, too large. Pre-1964 Niigata earthquake leveling surveys, previously used as confirmation of the dilatancy model, do not require dilatancy as an explanation and easily allow an alternative model with a subsurface density increase. Gravity is also not a dependable estimator of elevation change. But a combination of gravity with either leveling, if the dimensions of the distorted body are known or small, or geometric elevation measurements is essential for the determination of crustal density and strain changes. The 1965-1967 Matsushiro earthquake swarm leveling and gravity data show a significant dilatant strain of 0.6-1.8 $\times 10^{-4}$ if the proper model dimensions are used. This dilatant strain would be adequate to cause the observed drop in $V_{p} / V_{s}$, even if the crust were initially saturated prior to distortion. The combination of gravity, leveling, and the new geometric elevation measurements provides a useful parameter, gravitational potential, for the inversion of subsurface density distributions. Use of this parameter, defined as the free-air elevation anomaly, is illustrated for a nearly compensated mountain root structure and shows that this technique holds significant promise for the study of large, deep structures in the crust and upper mantle.
\end{abstract}

\section{INTRODUCTION}

In 1971 a joint earth physics program was begun between the California Institute of Technology and the Jet Propulsion Laboratory under the sponsorship of the National Aeronautics and Space Administration. One of its objectives is the development of new geodetic systems to solve the problems of crustal distortion measurements over long distances and their relationship to the occurrence of earthquakes. One outgrowth of this program has been the Aries (astronomical radio interferometric earth surveying) system [e.g., MacDoran, 1974; Ong et al., 1975a,b], which uses radio interferometry and currently measures station separations to $10-\mathrm{cm}$ three-dimensional accuracy for short base lines (less than $300 \mathrm{~km}$ ) and will be capable of $3-\mathrm{cm}$ or better accuracy over base lines of $0-1000 \mathrm{~km}$ and $10-\mathrm{cm}$ or better accuracy over intercontinental distances within a few years. Aries is one of a class of new geodetic techniques that involve either radio interferometry or laser ranging to aircraft, extragalactic radio sources, satellites, and the moon (see the review by Bender [1974]). The frame of reference of all of these systems is extraterrestrial. For example, the reference frame used by Aries is defined by the positions of extragalactic radio sources. These sources are so distant that they are a good physical approximation to an inertial coordinate system. A primary goal of any new geodetic system as it develops is a check of the system with conventional geodetic measurement techniques to verify accuracy whenever possible. However, analysis of the initial Aries field trials showed that a check of the geometric vertical component with leveling is not a simple task.

Elevation measurements as determined by geometric techniques, such as those described above, and by leveling are not directly comparable. This results from the fact that while a geometric measurement of elevation is direct, the interpretation of leveling data in terms of geometric elevations is density-model dependent, which is well known in geodesy. The

Copyright $(1976$ by the American Geophysical Union. difference between leveling and geometric ground shape has not been bothersome in the past because geodesists have been mainly interested in the direction and potential for flow of water which seeks to align its upper boundary to an equipotential surface, neglecting such dynamic considerations as ocean tides, wind, and Coriolis forces. Of course, after a correction for these forces, elevations as monitored by tidal gages are equivalent to leveling surveys because the ocean surface is used as the reference equipotential surface, the geoid. For the determination of subsurface strain and density changes, however, geophysicists require knowledge of the change in the geometric shape of the earth's surface. The ground shape estimates determined by leveling surveys may not be adequate for calculating those subsurface changes predicted by dilatancy models of preearthquake crustal distortion.

Figure 1 shows three basic types of geodetic elevation measurements that attempt to determine the shape of the ground surface, which in this example is always flat. Tiltmeters measure the angle between an equipotential surface and the ground surface. The process of leveling uses a reference equipotential surface and measures the distance between that surface and the ground surface. The measurement of distance from the reference equipotential surface to the ground surface involves gravity corrections (see the discussion by Heiskanen and Moritz [1967, p. 160]), and the resultant measurement, if it is referred to the geoid, is called the orthometric height. In this paper these corrections are assumed to be done perfectly, and heights measured by leveling are assumed to be true orthometric heights. Geometric methods measure the distance relative to some external frame of reference, represented here by a suspended star at great height, to the ground surface. In practice, both leveling and geometric methods measure elevation relative to another point on the ground surface, so that the elevation measurements shown in Figure 1 should be accompanied by measurements for some distant point with the same reference systems.

It should be noted that geometric heights can be calculated 

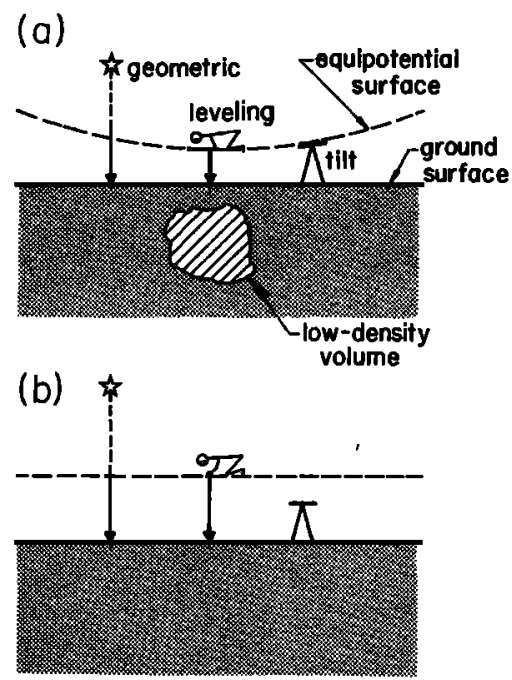

Fig. 1. Schematic drawing illustrating the relation between leveling, tilt, and geometric methods of measuring ground surface shape for a half space. (a) Lower-density volume inclusion. (b) Homogeneous density distribution. In part $a$, leveling and tilt measurements would indicate a bulge in the ground surface, whereas the geometric elevation measurement would indicate a flat surface. A transition from part $a$ to part $b$ would show a decrease in ground elevation from the leveling and tilt readings but no elevation change from a geometric measurement.

from leveling surveys through the use of Stokes' formula, which integrates gravity over the surface of the earth [Heiskanen and Moritz, 1967, p. 92]. However, the errors involved in the computation exceed $1 \mathrm{~m}$, even for idealized uniform gravity distributions [Heiskanen and Moritz, 1967, p. 276], and this technique appears to lack the resolution required for the problems discussed here. A further drawback of this method is that investigation of time-dependent changes in geometric elevation of a point would require the reoccupation of the detailed gravity net for each elevation determination.

Figure $1 a$ shows what will happen when an anomalous mass, here a lower-density volume in a homogeneous half space, is present just beneath the surface. Both the leveling and the tilt measurements, if they are taken at face value, indicate that the ground surface has a bulge over the anomaly, even though it is geometrically flat. The geometric measurement is unaffected by mass distributions and faithfully follows the geometric ground shape.

Now suppose that the low-density volume of Figure $1 a$ is eliminated by the transferral of mass from some distant source, so that the subsurface is homogeneous, as is shown in Figure $1 b$. The change in mass distribution will cause a decrease in elevation as measured by leveling and tilt, but the geometric measurement will register no change. Here again, tidal gages will give the same answer as leveling surveys if the tidal data can be corrected to account for dynamic forces.

So far, two classes of measurements have been discussed: those that depend on the shape of the ground surface and those that depend on the shape of the ground and equipotential surfaces. As shall be shown, a third extremely important class of geodetic measurements is gravity, which depends on the gradient of gravitational potential. Interpretation of subcrustal processes requires gravity, which is relatively easy to measure; gravity should be included in all studies of vertical crustal distortion.

Interpretation of vertical geodetic measurements has re- ceived considerable attention in the literature in relation to tectonic changes that occur in the crust both in the zone of earthquake preparation and in the zone of the earthquake and associated aftershocks [e.g., Tsubokawa et al., 1964; Whitcomb et al., 1973; Scholz et al., 1973; Castle et al., 1974; Nur, 1974; Kisslinger, 1975; Stuart and Johnston, 1975; Oliver et al., 1975]. Most of these studies have not estimated the model dependence of their interpretations of leveling, tilt, and gravity changes. In many cases, under reasonable assumptions, the model dependence is not severe; however, it will be shown that some types of data sets cannot be interpreted uniquely in terms of physical parameters of crustal materials. It is the purpose of this paper to analyze the model dependence of the various classes of vertical geodetic measurements, especially in relation to earthquake prediction and reasonable dilatancy models, and thereby to outline geodetic measurement procedures that provide the optimal means to further test physical models of the active tectonic medium. Finally, the use of the new geometric elevation data for inversion of the density structure of major tectonic features is illustrated with a simple model that approximates a nearly compensated mountain range.

\section{Model Dependence of Crustal Distortion MEasurements}

The gravitational attraction of a uniform circular disk will be used to calculate the effects of mass and volume changes beneath the earth's surface. All of the salient characteristics of the problem can be derived from the simple disk mass distribution or combinations of it. First, let us assume a simplified thin-disk model at the surface of the earth, as shown in Figure 2 , where $a$ is the radius of the disk ( $a \ll 6371 \mathrm{~km}$ ), $h$ is the initial thickness of the disk, $\rho_{1}$ is the initial density of the disk, $\rho_{2}$ is the final density of the disk, and $\epsilon$ is the incremental thickness change of the disk and also the elevation change at the surface of the disk; cgs units will be used throughout unless it is otherwise stated. The potential and the gravitational attraction at a point on the positive $z$ axis due to the thin disk alone are well known [Ramsey, 1959, p. 35]:

$$
\begin{aligned}
& V=2 \pi k \rho_{1} h\left[\left(z^{2}+a^{2}\right)^{1 / 2}-z\right] \\
& G=-\frac{\partial V}{\partial z}=2 \pi k \rho_{1} h\left[1-z\left(z^{2}+a^{2}\right)^{-1 / 2}\right]
\end{aligned}
$$

where $V$ is potential, $G$ is gravitational acceleration, $|\Delta V|=$ $-\partial V / \partial z, k$ is the gravitational constant (equal to $6.673 \times 10^{-8}$ $\mathrm{cm}^{3} \mathrm{~g}^{-1} \mathrm{~s}^{-2}$ ), and $z$ is the vertical coordinate. If measurements of these gravitational quantities are made at the earth's surface, then $z / a$ is small, and relations ( 1 ) are approximated by

$$
\begin{aligned}
& V_{1}=2 \pi k \rho_{1} h a+V_{0} \\
& G_{1}=2 \pi k \rho_{1} h+G_{0}
\end{aligned}
$$

where $V_{0}$ and $G_{0}$ are the potential and gravity, respectively, due to that part of the earth exclusive of the disk. The second of relations (2) is the familiar infinite slab gravity formula. However, there is no equivalent expression for the potential $V_{1}$ because it is readily apparent that $V_{1}$ is dependent on $a$. Herein lies the basic difference between potential-dependent quantities, such as leveling elevations, and those that depend on the spatial derivative of the potential, such as gravitational attraction and tilt. Gravitational contributions from distant mass distributions are more significant to the potential field than to the attraction field, as is obvious from the gravitational 


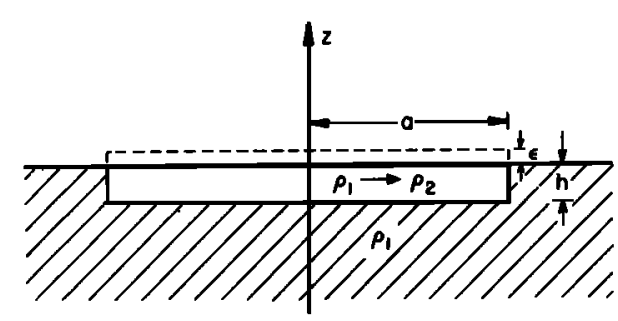

Fig. 2. Disk model used for gravitational calculations, where $a$ is the radius of the disk, $h$ is the initial thickness of the disk, $\rho_{1}$ is the initial density of the disk, $\rho_{2}$ is the final density of the disk, and $\epsilon$ is the incremental thickness change of the disk and also the elevation change at the earth's surface.

relations for a point mass $m$ at a distance $r$ from an observer: $V=k m / r$, and $G=k m / r^{2}$.

Now suppose in the simplified disk model that the density changes to $\rho_{2}$ and an increment in volume changes the thickness of the disk and the elevation of the ground surface by $\epsilon$. The gravitational relations at the upper surface of the disk are now

$$
\begin{aligned}
& V_{2}=2 \pi k \rho_{2}(h+\epsilon) a-\alpha \epsilon+V_{0} \\
& G_{2}=2 \pi k \rho_{2}(h+\epsilon)-\beta \epsilon+G_{0}
\end{aligned}
$$

where $\alpha=981 \mathrm{~cm} \mathrm{~s}^{-2}$ and $\beta=3.08 \times 10^{-8} \mathrm{~s}^{-2}$ are the free-air gradients of potential and gravity, respectively. The difference of (2) and (3) provides what an observer would see as changes at the ground surface in the middle of the disk:

$$
\begin{aligned}
& \Delta V=V_{2}-V_{1}=2 \pi k \Delta \rho h a+\epsilon\left(2 \pi k \rho_{2} a-\alpha\right) \\
& \Delta G=G_{2}-G_{1}=2 \pi k \Delta \rho h+\epsilon\left(2 \pi k \rho_{2}-\beta\right)
\end{aligned}
$$

where $\Delta \rho=\rho_{2}-\rho_{1}$.

Leveling surveys across the disk before and after the change directly measure the potential change above the center of the disk relative to distant values and, to first order, calculate the orthometric elevation change by the formula

$$
\epsilon^{\prime}=-\Delta V / \alpha
$$

But from (4) the geometric change is

$$
\epsilon=\epsilon^{\prime}\left[1+\left(\rho_{2}+\frac{\Delta \rho h}{\epsilon^{\prime}}\right)\left(\frac{2 \pi k}{\alpha / a-2 \pi k \rho_{2}}\right)\right]
$$

with the use of (5).

An equivalent expression for geometric elevation change related to a gravity change $\Delta G$ from (4) is

$$
\epsilon=(\Delta G-2 \pi k \Delta \rho h) /\left(-\beta+2 \pi k \rho_{2}\right)
$$

If both $\Delta G$ and $\Delta V$ are measured, the combination of relations (4) gives for geometric elevation change

$$
\epsilon=\frac{\epsilon^{\prime} \alpha / a+\Delta G}{\alpha / a-\beta}
$$

Thus by using the gravity we have eliminated all dependence on thickness and density for the disk model, but the horizontal characteristic dimension, radius $a$, remains as an independent variable that must be known or assumed before the geometric elevation change can be calculated.

An important parameter that is used as a measure of mass change under a site is the ratio of the change in gravity to the change in geometric elevation, $\Delta G / \epsilon$, which we define as the distortion gravity gradient $\gamma$. This quantity is sometimes sim- ply called the gravity gradient, but that term is not used here in order to avoid confusion with its more common usage for the instantaneously measured gradient. Studies usually have approximated $\gamma$ by $G / \epsilon^{\prime}=\gamma^{\prime}$. From (8), $\gamma$ can be written as

$$
\gamma=\gamma^{\prime}\left(1-\frac{\gamma^{\prime}+\beta}{\gamma^{\prime}+\alpha / a}\right)
$$

Equations (6)-(8) are useful for estimating the model dependence of the use of leveling and/or gravity to determine both geometric elevation changes and the distortion gravity gradient, provided that the measurements can be considered to be over the central part of a shallow, thin structure, a good approximation for many of the cases encountered.

For example, consider precursory evidence for shallow earthquakes. Anderson and Whitcomb [1975] developed from several lines of evidence that the characteristic dimension of precursory anomalous areas associated with at least some types of earthquakes is represented by the relation

$$
\log L(\mathrm{~km})=0.26 M+0.46
$$

where $L$ is the approximate horizontal dimension of the anomalous zone and $M$ is the earthquake magnitude. For an $M=$ 6.4 earthquake like the 1971 San Fernando, California, event, $L$ is $133 \mathrm{~km}$, giving an $a$ of $66 \mathrm{~km}$. Castle et al. [1974] have interpreted leveling data obtained prior to the San Fernando earthquake and have found a change in $\epsilon^{\prime}$ of at least $10 \mathrm{~cm}$ near the epicentral area in the 10 years prior to the earthquake. Anderson and Whitcomb $[1973,1975]$ have calculated that density changes during a dilatancy cycle can easily be $0.001 \mathrm{~g} / \mathrm{cm}^{3}$ (a change of about $0.04 \%$ ), and the velocity data of Whitcomb et al. [1973] and Anderson and Whitcomb [1975] require a thickness of the dilatancy zone of the order of $10 \mathrm{~km}$. With $\Delta \rho$ $=-0.001 \mathrm{~g} / \mathrm{cm}^{3}, h=10 \mathrm{~km}$, and $\rho_{2}=2.7 \mathrm{~g} / \mathrm{cm}^{3},(6)$ gives a geometric elevation change of $7.23 \mathrm{~cm}$. The associated gravity change from (4) is $-0.433 \mathrm{mGal}$. Thus for this particular set of model parameters the leveling estimate of elevation change is high by $2.77 \mathrm{~cm}$, or $38 \%$. This can be an important source of uncertainty if one is trying to calculate volume and mass changes in the crust. What about the effect of the uncertainty of $a$ ? Some estimates of the magnitude of the San Fernando earthquake, especially at European stations, were $M=6.7$. From (10) this gives $a=79 \mathrm{~km} . \Delta G$ would remain unchanged by this modification of the model, and the geometric elevation change would then be, from $(8), \epsilon=6.68 \mathrm{~cm}$. Thus a reasonable $20 \%$ increase in $a$ gives a $0.55 \mathrm{~cm}$, or $8 \%$, decrease in the calculated geometric elevation change, even if both $\Delta G$ and $\epsilon^{\prime}$ are measured. However, the combination of gravity and leveling is a substantial improvement over either survey alone because it eliminates dependence on both $\Delta \rho$ and $h$ for most models, as can readily be seen by a comparison of (6), (7), and (8).

The geometric distortion gravity gradient $\gamma$ for the first San Fernando model above is $-5.99 \times 10^{-5} \mathrm{~s}^{-2}$, whereas the gradient calculated from leveling, $\gamma^{\prime}$, is $-4.33 \times 10^{-5}$, a $28 \%$ difference. For the second model with the increased radius, $\gamma$ is $-6.48 \times 10^{-5} \mathrm{~s}^{-2}$, giving a $33 \%$ difference between gradients determined by geometric and leveling elevations. The difference between the actual distortion gravity gradients for the two models is $8 \%$.

A useful way to look at the relation of $\Delta G, \epsilon^{\prime}$, and $\epsilon$ for a given model over a range of one of the model parameters (of which $\Delta \rho$ is one of the more significant) is in a combined graph, here termed a gravity-leveling-elevation (GLE) plot. Figure 3 illustrates the previous San Fernando $a=66 \mathrm{~km}$ 


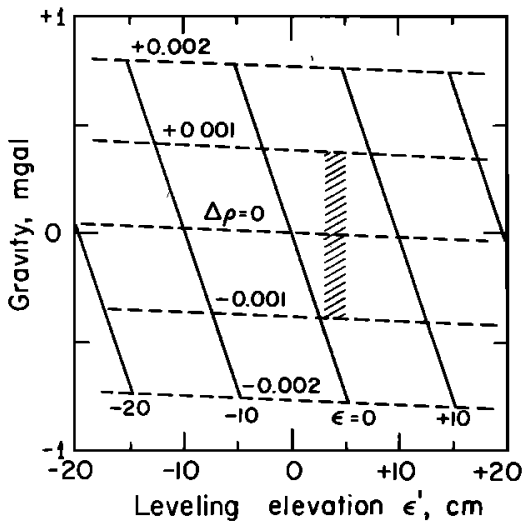

Fig. 3. GLE (gravity-leveling-elevation) plot for a specific subsurface model geometry. Here the model consists of a buried disk of 66-km radius, 10-km thickness, and 5-km center depth, with an observation point on the earth's surface at the central axis. Gravity and leveling elevation form the axes, and geometric elevation and density contours occupy the interior. The hatched area shows that the 3- to 5$\mathrm{cm}$ leveling anomaly observed before the Niigata earthquake does not necessarily mean that dilatancy occurred and a subsurface density increase is equally likely from the leveling data.

model in such a plot. $\Delta G$ is the vertical axis, $\epsilon^{\prime}$ is the horizontal axis (as is the usual method of plotting changes in gravity and leveling data), and contours of equal $\epsilon$ and equal $\Delta \rho$ occupy the interior. This mode of presentation for the relations between a model's geodetic parameters becomes more convenient as one leaves the simplicity of a single disk model and the assumption of being above the central part of the distorted body.

More complicated distortion gravitational effects, those of cylinders, for example, can be estimated to a desired accuracy by considering the linear sum of multiple thin-disk models. This technique also allows the simple calculation for observation points off the axis of symmetry. The gravitational potential of a thin circular disk is [Ramsey, 1959, p. 133]

$$
\begin{aligned}
& V_{r<a}=2 \pi k \rho h\left(a-r P_{1}\right.+\frac{1}{2} \frac{r^{2}}{a} P_{2} \\
&\left.-\frac{1 \cdot 1}{2 \cdot 4} \frac{r^{4}}{a^{3}}+\frac{1 \cdot 1 \cdot 3}{2 \cdot 4 \cdot 6} \frac{r^{6}}{a^{5}} P_{6}-\ldots\right) \\
& V_{r>a}=2 \pi k \rho h\left(\frac{1}{2} \frac{a^{2}}{r}-\frac{1 \cdot 1}{2 \cdot 4} \frac{a^{4}}{r^{3}} P_{2}\right. \\
&\left.+\frac{1 \cdot 1 \cdot 3}{2 \cdot 4 \cdot 6} \frac{a^{a}}{r^{5}} P_{4}-\ldots\right)
\end{aligned}
$$

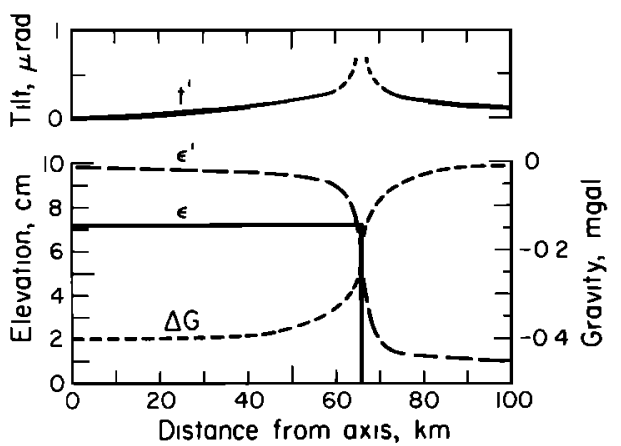

Fig. 4. Changes in gravity $G$, geometric elevation $\epsilon$, elevation from leveling $\epsilon^{\prime}$, and apparent tilt $t^{\prime}$ as measured at the earth's surface as a function of radius from the axis of the disk model of Figure 3. A density change of $-0.001 \mathrm{~g} / \mathrm{cm}^{3}$ is assumed. where $\rho$ is now either the change in density of the buried disk or the density of the disk elevated above the surface by an amount $\epsilon$. The $P$ are Legendre polynomials of the first kind and are functions of $\mu=\cos \theta$, and the observation point is at coordinates $(r, \theta) ; \theta$ is the angle measured from the positive vertical axis, and $r$ is the radial distance from the center of the disk. The gravitational attraction of the disk is then $\mathbf{G}=$ $-\nabla V$, or

$$
\begin{aligned}
& {\left[\begin{array}{c}
G_{r} \\
G_{\theta}
\end{array}\right]_{r<a}=2 \pi k \rho h} \\
& {\left[\begin{array}{c}
\left(\rho_{1}-\frac{r}{a} P_{2}+\frac{1 \cdot 1}{2} \frac{r^{3}}{a^{3}} P_{4}-\frac{1 \cdot 1 \cdot 3}{2 \cdot 4} \frac{r^{5}}{a^{5}} P_{6}+\cdots\right) \\
\frac{\partial \mu}{\partial \theta}\left(\frac{\partial P_{1}}{\partial \mu}-\frac{1}{2} \frac{r}{a} \frac{\partial P_{2}}{\partial \mu}+\frac{1 \cdot 1}{2 \cdot 4} \frac{r^{3}}{a^{3}}-\frac{1 \cdot 1 \cdot 3}{2 \cdot 4 \cdot 6} \frac{r^{5}}{a^{5}} \frac{\partial P_{6}}{\partial \mu}+\cdots\right)
\end{array}\right]}
\end{aligned}
$$$$
\left[\begin{array}{l}
G_{r} \\
G_{\theta}
\end{array}\right]_{r>a}=2 \pi k \rho h
$$$$
\cdot\left[\begin{array}{c}
\left(\frac{1}{2} \frac{a^{2}}{r^{2}}-\frac{1 \cdot 1 \cdot 3}{2 \cdot 4} \frac{a^{4}}{r^{4}} P_{2}+\frac{1 \cdot 1 \cdot 3 \cdot 5}{2 \cdot 4 \cdot 6} \frac{a^{6}}{r^{6}} P_{4}-\cdots\right) \\
\frac{\partial \mu}{\partial \theta}\left(\frac{1 \cdot 1}{2 \cdot 4} \frac{a^{4}}{r^{4}} \frac{\partial P_{2}}{\partial \mu}-\frac{1 \cdot 1 \cdot 3}{2 \cdot 4 \cdot 6} \frac{a^{6}}{r^{6}} \frac{\partial P_{4}}{\partial \mu}+\cdots\right)
\end{array}\right]
$$

The change in potential and gravity is computed from a linear sum of the effects of thin disks that have undergone a density change, plus the effect of the elevated disk, plus free-air corrections due to a displacement of the observation point in the earth's gravitational field in parallel with the development leading to the terms in (4). The difference in measured gravity is

$$
\Delta G=\left|\mathbf{G}+\mathbf{G}_{\mathbf{0}}\right|-\left|\mathbf{G}_{\mathbf{0}}\right|
$$

where $\mathbf{G}_{0}$ is the gravity vector before deformation and the vertical bars indicate absolute values. If $G_{x}$ is defined as the outward horizontal component of the gravitational attraction, then the apparent tilt $t^{\prime}$ due to the changed gravitational field alone can be written as

$$
t^{\prime}=-G_{x} / \alpha
$$

where a positive tilt indicates an apparent tilt of the ground down away from the central axis.

Figure 4 shows calculations from (12) for changes in gravity, geometric elevation, elevation from leveling, and apparent tilt for the $a=66 \mathrm{~km}$ San Fernando model as measured at the earth's surface. Note the effect as an observer approaches the edge of the disk from inside or outside. Figure 5 shows the geodetic relations in a GLE plot for the same model variations as are shown in Figure 3 except that the observation point is now $5 \mathrm{~km}$ outside the edge of the distortion region. Here no geometric elevation change takes place, but both gravity and leveling changes are observed.

The above calculations point out the difficulty in interpretation of leveling or gravity alone for use in confirming earthquake precursory models such as those which involve dilatancy. Accurate estimation of the volume change during crustal distortion is extremely important in the development of a physical model of the earthquake process, and this is most directly estimated from geometric horizontal and vertical displacements of the earth's surface. A combination of leveling and gravity data reduces the model dependence significantly 
but is still dependent on the assumed extent of the altered body. Important leveling data prior to the 1964 Niigata earthquake were obtained by Tsubokawa et al. [1964] that indicated a deviation in the long-term elevation change rate starting approximately 6-10 years before the event; the anomalous change is about $3-5 \mathrm{~cm}$. The model for the GLE plot of Figure 3 is a conservative approximation of the dimensions for the pre-Niigata anomaly. The hatched area in the figure shows that the observation of $\epsilon^{\prime}=3-5 \mathrm{~cm}$ can correspond to a geometric elevation change of $\epsilon=0-2 \mathrm{~cm}$ or $\epsilon=6-8 \mathrm{~cm}$, depending on whether the density change is from -0.001 or $+0.001 \mathrm{~g} / \mathrm{cm}^{3}$, ,respectively. Whitcomb et al. [1973] and Scholz et $\ddot{a}$. [1973] have used the Niigata leveling data as confirmation of dilatancy prior to the earthquake, but Figure 3 shows that a density increase is equally likely. Thus in the absence of gravity data, dilatancy is not required, and a reasonable alternative model is an increase in horizontal compressive stress in the area, causing an increase in both density and elevation. These factors emphasize that before vertical geodetic parameters can be used to confirm physical models of the earthquake process, the uniqueness of their interpretation must be carefully examined.

Recent surveys in which both $\Delta G$ and $\epsilon^{\prime}$ have been measured in relation to earthquakes are those for the Matsushiro swarm in Japan (Kisslinger [1975] gives a review of the data) and the San Fernando earthquake in California [Oliver et al., 1975]. If the disk model has no density change during distortion, the distortion gravity gradient $\gamma$ is $-1.95 \times 10^{-6} \mathrm{~s}^{-2}$, termed the Bouguer gradient. If the density change is such that no horizontal mass transfer takes place, the gradient is $-3.08 \times 10^{-6}$ $\mathrm{s}^{-2}$, which is the free-air gravity gradient. In both cases the difference between the apparent and the actual values of gradient and elevation is less than $1 \%$ for reasonable ranges of $a$ from (9) for the data of Oliver et all. They show that the best fit to the San Fernando data is the Bouguer gradient, implying $\Delta \rho=0$, but the San Fernando data were not taken during the time of velocity decrease [Whitcomb et al., 1973] and are presumably related to the difference between predilatancy and postdilatancy deformation.

The Matsushiro data pertain only to the time of the earthquake swarm, and preswarm deformation is unknown. A good review of the extensive gravity and leveling data gathered by Japanese scientists is given by Kisslinger [1975]. Nur [1974] and Kisslinger [1975] argue that the geodetic data plus correla-

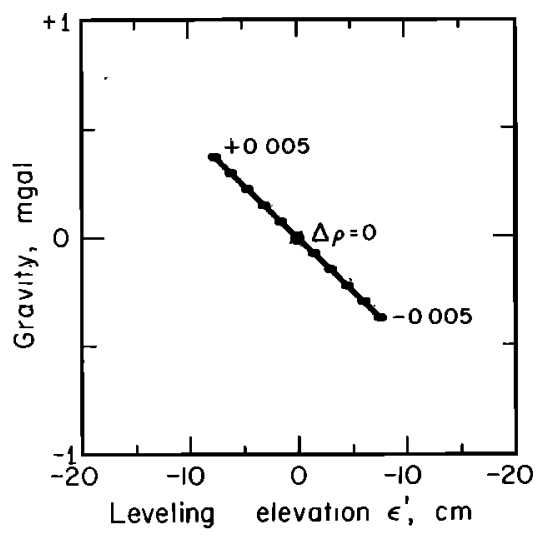

Fig. 5. GLE plot for the same model variations as are shown in Figure 3 except that the observation point is now $5 \mathrm{~km}$ outside the edge of the distortion region at a radius of $71 \mathrm{~km}$. Here no geometric elevation change takes place, but both gravity and leveling changes are observed.

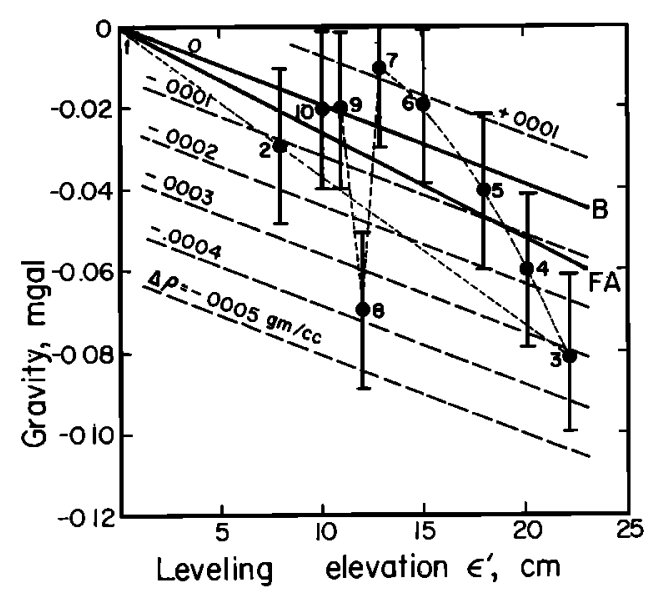

Fig. 6. GLE plot of Matsushiro gravity and leveling data from Kisslinger [1975] with \pm 0.02 -mGal error bars. The times of observation are as follows: point 1, October 1965; 2, June 1966; 3, September 1966; 4, October 1966; 5, December 1966; 6, April 1967; 7, November 1967; 8, March 1968; 9, December 1968; and 10, December 1968. A model with $a=5 \mathrm{~km}$ and thickness of $5 \mathrm{~km}$ was used to compute the free-air distortion gravity gradient $F A\left(\gamma=-0.261 \times 10^{-5} \mathrm{~s}^{-2}\right)$. The Bouguer gradient $B$ is the same for all models $\left(\gamma=-0.196 \times 10^{-3}\right.$ $\mathbf{s}^{-2}$ ). Data that fall above $F A$ indicate horizontal mass flow into the region, and those that fall below $F A$ indicate mass flow outward in relation to point 1 . The contours indicate a subsurface density change of -0.00015 to $-0.00048 \mathrm{~g} / \mathrm{cm}^{3}$ for point 3 , which is sufficient to cause a lowering of $\boldsymbol{P}$ wave velocity even if the crust were initially saturated.

tion of spring flow with seismic energy release indicate that the swarm phenomena strongly favor the dilatancy-fluid diffusion model. Nur's analysis unfortunately is based on erroneous calculations for the distortion gravity gradients. Stuart and Johnston [1975] favored a shallow magma intrusion as an explanation of the swarm, showing a strong correlation of the spring flow with rainfall, and concluded that the geodetic data fall within bounds of both the intrusion and the dilatancy hypotheses. Of these analyses, only the analysis by Kisslinger made an estimate of the model dependence of the distortion gravity gradient for what is accepted as the characteristic dimerision of the affected volume, a disk with radius and depth of about $5 \mathrm{~km}$. Figure 6 shows the data from Kisslinger in a GLE plot (for this model, $\epsilon^{\prime}$ is within $1 \%$ of $\epsilon$, and the elevation contours are omitted). The distortion free-air gradient for the model is $\gamma=-0.261 \times 10^{-5} \mathrm{~s}^{-2}$; if the radius were increased to $10 \mathrm{~km}$, the gradient would be $\gamma=-0.282 \times 10^{-5} \mathrm{~s}^{-2}$. The Bouguer gradient is virtually the same for all models $(\gamma=$ $\left.-0.196 \times 10^{-5} \mathrm{~s}^{-2}\right)$, and the Bouguer water gradient, on the assumption that the cracks fill with water, is approximately one-third the distance from the free-air line to the Bouguer line. The error bars are the same as those shown by Stuart and Johnston. It can be seen that the data point for September 1966 falls significantly below the distortion free-air gradient for the 5-km-radius model, in contrast to Stuart and Johnston's conclusions. If one were to adopt the intrusion model of Stuart and Johnston [1975, Figure 3], the reduced model dimensions would make the computed distortion free-air gradient shallower and even further from the data point. In addition, the data of Figure 6 are from well on the flanks of the anomalous uplift. Examination of the edge effect on the gradient of Figure 4 shows qualitatively that the distortion free-air gradient is less negative near the edge of the anomalous body. Thus unless a case can be made that the radius of the anomalous body is much larger than $5 \mathrm{~km}$, for example, 10 
$\mathrm{km}$, the single point of September 1966 is significantly below a reasonable estimate of the distortion free-air gradient; and if this datum is acceptéd, horizontal mass movement (loss) had to take place during the uplift part of the cycle. The subsurface density decrease from Figure 6 would be in the range 0.00015-0.00048 $\dot{\mathrm{g}} / \mathrm{cm}^{3}$.

The dilatancy modet was originally proposed because it was the only reasonable explanation of anomalous values of the seismic velocity ratio $V_{p} / V_{s}$ prior to earthquakes [ $N u r, 1972$ ]; shown to be due mainly to a drop in $P$ wave velocity [Whit comb et al., 1973]. The only $V_{p} / V_{s}$ data that are available for the Matsushiro swarm have been presented by Ohtake [1973] but have not been discussed by the above authors. Ohtake's data show a drop in averaged values of $V_{p} / V_{s}$ during the entire swarm. Whitcomb et al. [1973] proposed a dilatancy-diffusion model that allowed the initial conditions of a saturated crust. Anderson and Whitcomb $[1973$; 1975] estimated the fluid pore pressure drop required to achieve a slgnificant drop in $V_{p}$ starting from ambient saturated crustal conditions. With the use of these calculations and the equation of state of water it is possible to estimate the dilatant volumetric strain required to cause a significant $V_{p}$ decrease, when a given initial saturated pore pressure and no fluid flow into the volume are assumed. Figure 7 shows the required dilatant strain as a function of initial pore pressure with the assumption of an initial porosity of $0.1 \%$. The crosshatched area corresponds to approximate crustal conditions at Matsushiro and shows that less than 0.8 $\times 10^{-4}$ dilatant strain is required, compared with the 0.6-1.8 $\times$ $10^{-4}$ strain calculated from the density change in Figure 6 . If the model is changed to one with radius $a=10 \mathrm{~km}$, the corresponding strain will be $0.4-1.4 \times 10^{-4}$. The effect of nearness to the edge of the anomalous mass will increase these strain estimates. Thus the geodetic data indicate sufficient strain to start with a saturated crust prior to the swarm and still get $V_{p}$ reductions, a finding which does not confirm but certainly allows the variations reported by Ohtake.

\section{GLE INVERSION FOR CRUST AND UPPER Mantle Strúcture}

Geometrical elevation measurements provide a new parameter for the inversion of subsurface density distributions. This

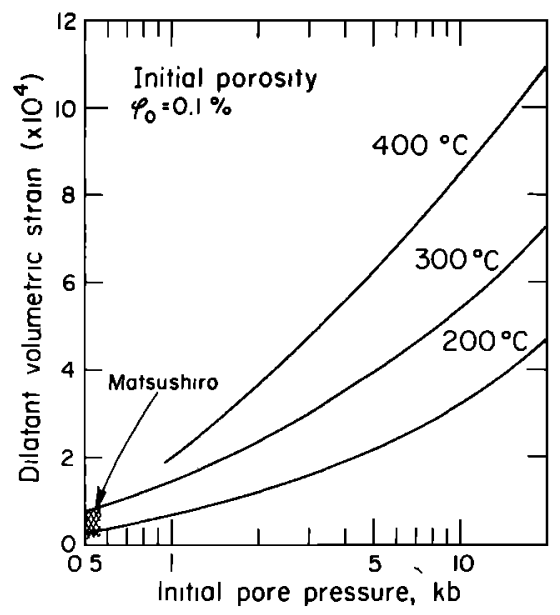

Fig. 7. Dilatant volumetric strain required to cause significant $V_{p}$ decrease as a function of initial water pore pressure, an initial porosity of $0.1 \%$ and no water flow being assumed. The isotherms are calculated from data of Kennedy and Holser [1966]. Hydrostatic pressure calculated for the Matsushiro model shows that less than $0.8 \times 10^{-4}$ dilatant strain is required, compared with the $0.6-1.8 \times 10^{-4}$ observed from Figure 6.
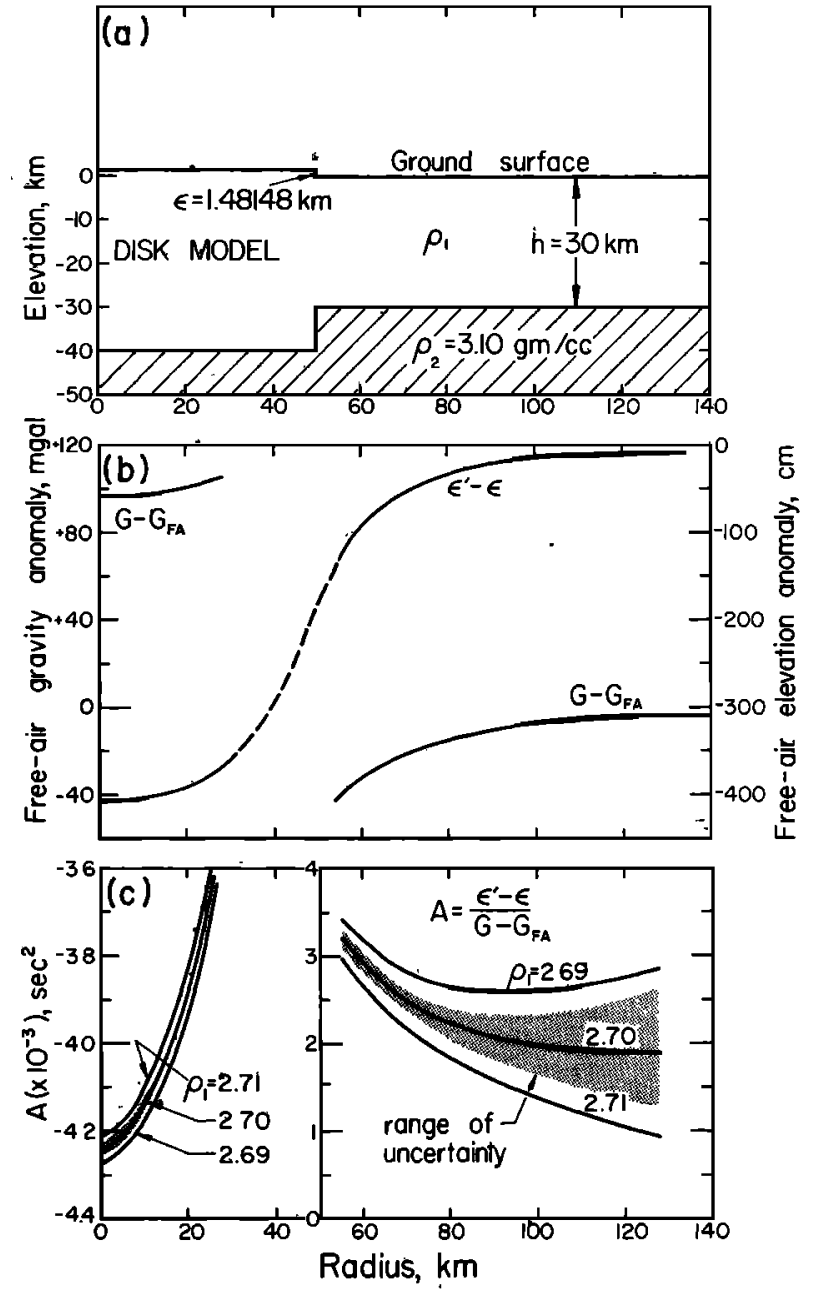

Fig. 8. (a) Cross section of a disk model used to approximate the characteristic parameters of a large mountain range and its associated compensating root extending $10 \mathrm{~km}$ below the nominal lower surface of the crust. If the crustal density is $2.70 \mathrm{~g} / \mathrm{cm}^{3}$, molintains at elevation $1.48148 \mathrm{~km}$ are exactly compensated. (b) Surface observations of the free-air gravity anomaly $G-G_{F A}$ and the free-air elevation anomaly $\epsilon^{\prime}$ $-\epsilon$ as a function of radius from the axis of the model. Note that the difference between leveling and geometric elevation is more than $4 \mathrm{~m}$ at the center of the disk. (c) Parameter $A=\left(\epsilon^{\prime}-\epsilon\right) /\left(G-G_{F A}\right)$, as observed at the surface with a crustal density for a compensated model, $2.70 \mathrm{~g} / \mathrm{cm}^{8}$; an overcompensated model, $2.69 \mathrm{~g} / \mathrm{cm}^{3}$; and an undercompensated model, $2.71 \mathrm{~g} / \mathrm{cm}^{3}$. The e'ror estimates are plotted for the compensated model and assume \pm 5 -cm uncertainty in $\epsilon^{\prime}-\epsilon$. In practice, $A$ would be most useful where it is least variable with radius, i.e., beyond the edge of the disk and perhaps at the center.

parameter is the gravitational potential along the earth's surface which, when it is used with gravity, the gradient of the potential, gives added power to resolve the classic nonuniqueness of gravitational inversion problems. In gravity, various anomalies have been defined to remove the effect of known or assumed model parameters; the simplest of these that involves no assumptions of model densities is the free-air gravity anomaly, which makes only a free-air correction for the elevation of the observation póint, written here as $G-G_{F A}$. The equivalent anomaly for the potential in terms of the observables discussed above is defined here as the free-air elevation anomaly, $\epsilon^{\prime}-\epsilon$. The usefulness of this anomaly is of course contingent on its size, which is model dependent, and on the accuracy with which it can be measured. As was shown above, models of dimensions of the order of $10 \mathrm{~km}$ have relatively small elevation anomalies for reasonable density contrasts. 
Measurements, however, are now approaching accuracies such that they can be useful for study of bodie's of dimensions significantly larger than $10 \mathrm{~km}$; a good example is a mountain range like the Sierra Nevada range in the western United States. Oliver et al. [1961] fitted a two-dimensional model to gravity data over the Sierra Nevada range. 'In order to calculate the effect of the structure of the range on our anomalies defined above the crustal structure of Oliver et al. is approximated by the disk model shown in Figure $8 a$. This model has a $10-\mathrm{km}$ root extending below a nominal $30-\mathrm{km}$ crust. The mountains are exactly compensated by the root if the crustal density is $2.70 \mathrm{~g} / \mathrm{cm}^{3}$. Figure $8 b$ shows the variation of the freeair gravity and elevation anomalies, $G-G_{F A}$ and $\epsilon^{\prime}-\epsilon$, measured at the earth's surface of the model. Note that the difference between leveling and geometric elevation is more than $4 \mathrm{~m}$ at the center of the disk.

It was determined above that the ratio of gravity to elevation change, the distortion gravity gradient, was sensitive to the subsurface mass change. The equivalent concept applied to tectonics of large mountain roots translates into mass compensation, i.e., whether the elevated range is compensated by an equivalent lack of mass at depth in a crustal root. Because the values in Figure $8 b$ show that relative uncertainties in $\epsilon^{\prime}-\epsilon$ are mych greater than those in $G-G_{F A}$, a more stable parameter is the inverse of the gravity gradient:

$$
A=\left(\epsilon^{\prime}-\epsilon\right) /\left(G-G_{F A}\right)
$$

Figure $8 c$ shows the parameter $A$ as observed at the surface with a crustal density for a compensated model, $2.70 \mathrm{~g} / \mathrm{cm}^{\mathrm{s}}$; an overcompensated model, $2.69 \mathrm{~g} / \mathrm{cm}^{3}$; and an undercompensated model, $2.71 \mathrm{~g} / \mathrm{cm}^{3}$. In terms of the depth of the root these calculations are equivalent to \pm 37 -m deviation from the compengated model depth. The error bars shown for the compensated model assume a \pm 5 -cm uncertainty in $\epsilon^{\prime}-\epsilon$; this will be mainly limited by uncertainties in $\epsilon^{\prime}$. It is seen that the relatively minor dgiviations from compensation are resolvable even if the error is doubled to $\neq 10 \mathrm{~cm}$. In practice, the parameter $A$ would be most useful where it is least variable with radius, beygind the edge of the disk and perhaps at the center. As a further example of the sensitivity of $A$ to depth variations of the model of Figure $8 a$ the elevated mountain range is held compensated, but the nominal çrustal depth $h$ is allowed to vary with an observation point at $55-\mathrm{km}$ radius. Figure 9 shows that with an assumed \pm 5 -cm error in $\epsilon^{\prime}-\epsilon$ the g̣rustal depth is resolvable to within $\pm 3 \mathrm{~km}$.

\section{Conclusions}

Vertical geodesy is undergoing a revolution because of two factors. First, new preçise three-dimensional position-measuring techniques over very long distances provide important data previously unavailable for geophysical investigations. These techniques use extraterrestrial objects for reference, such as aircraft, satellites, the moon, or extragalactic radio sources, and employ laser ranging or radio interferometry for measurement. One such portable system, Aries, which uses radio interferometry, is currently measuring $0-$ to $300-\mathrm{km}$ separations to within $10 \mathrm{~cm}$ for three dimensions, or 3 parts in $10^{7}$. It is estimated that within a few years the accuracies will be $3 \mathrm{~cm}$ from 0 to $1000 \mathrm{~km}$ and $10 \mathrm{~cm}$ at intercontinental distances. Importantly, the vertical component of these techniques, the geometric elevation measurement, is a new class of data that is distinct from leveling in that the latter depends on the density structure of the earth for interpretation.

Second, recent developments in earthquake tectonic theory

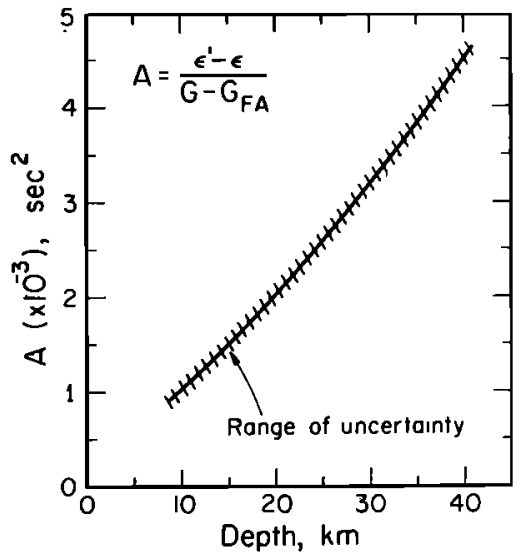

Fig. 9. Parameter $A$ observed at $55-\mathrm{km}$ radius for the compensated model of Figure $8 a$ except that the crustal thickness $h$ is allowed to vary. With an assumed $\pm 5-\mathrm{cm}$ uncertainty in $\epsilon^{\prime}-\epsilon$ the crustal depth is resolvable to within $\pm 3 \mathrm{~km}$.

predict crustal distortions that have significant impact on the conventional assumptions used to interpret gravity, tilt, and leveling data. For the distortions believed to be associated with large earthquakes these assumptions are violated. For example, if reasonable parameters for the dilatancy model are applied to the 1971 San Fernando, California, earthquake, the 10-cm elevation changes reported from preearthquake leveling data are about $3 \mathrm{~cm}$, or $40 \%$, higher than the geometric elevation changes. Apparent tilts near the edges of this model are of the same order as solid earth tide tilts. Leveling data prior to the 1964 Niigata, Japan, earthquake have been widely used as confirmation of preearthquake dilatancy. However, in the absence of gravity surveys, dilatancy is not required, and a reasonable alternative model with a density increase is easily allowed by the leveling data. These factors emphasize that before vertical geodetic parameters can be used to confirm physical models of the earthquake process, the uniqueness of their interpretation must be carefully examined.

Future development of physical models for the earthquake zone of preparation will depend on an accurate knowledge of its distortion and associated density change. In order to achieve this, certain minimum requirements for geodetic data must be met. Gravity must be measured simultaneously with one of the elevation measurement techniques through the time of distortion. A change in any one of the vertical geodetic measurements alone will indicate a physical change within the earth, but interpretation of the change is difficult and involves too many model assumptions. Gravity and leveling are adequate if the physical dimensions of the distorted body are small. A relation like (8) can be used to evaluate with given or estimated changes in gravity and leveling whether uncertainties in the physical dimension of the model are unacceptable for the estimation of volumetric parameters from geometric elevation.

The accuracy of leveling over large distances is difficult to assess because it involves assumptions of the error properties. In this author's opinion, if the possible dimensions of the distortion model exceed about $60 \mathrm{~km}$, geometric techniques with accuracies of $3 \mathrm{~cm}$ begin to be able to resolve the uncertainties in geometric elevation that remain with only first-order leveling and gravity surveys in active tectonic areas. If crustal distortion occurs with time scales short with respect to reasonable repeat times for first-order leveling surveys over large distances, for example, within months over distances of hun- 
dreds of kilometers, only geometric techniques are reasonably capable of giving both the accuracy and the time resolution necessary to monitor the crustal distortion. For example, monthly monitoring of the three-dimensional relative position of stations separated by $200 \mathrm{~km}$ in southern California was conducted by the Aries project in the first half of 1975 in order to investigate the possible crustal distortion related to a seismic velocity anomaly in the area. The distortion zone for the 1965-1967 Matsushiro earthquake swarm probably satisfies the size condition above, and the extensive leveling and gravity data obtained during the swarm have been widely discussed. Analysis of the data with a reasonable density model taken into account shows that the data fall significantly below the free-air gradient and imply a dilatant strain in the range $0.6-1.8 \times 10^{-4}$. This strain, if it is interpreted as being all void increase in the subsurface rocks, is adequate to cause the reported drop in $V_{p} / V_{q}$, even if the crustal rocks were saturated with water at hydrostatic pore pressure just prior to distortion.

The ideal geodetic survey would incorporate all three techniques, gravity, leveling, and geometric elevation, because the last technique provides a new parameter for the inversion of subsurface density distributions. This parameter is the gravitational potential, which, when it is used with gravity, gives added power to resolve the classic nonuniqueness of gravitational inversion problems. The practical use of the potential parameter is limited to bodies of dimensions of the order of 50 $\mathrm{km}$ or larger. One of this large class of tectonic structures is a mountain range and its associated uncertainties of compensation and root extent. Resolution calculations for a Sierra Nevada sized structure shows that variations of density of 0.01 $\mathrm{g} / \mathrm{cm}^{3}$ or depth of $37 \mathrm{~m}$ of the root from a compensated model cause measurable changes of the potential parameter. Because the potential field varies as the inverse of the distance from the attracting mass, whereas gravity varies as the inverse squared, the combination of the two data types holds significant promise for the study of large, deep structures in the crust and upper mantle.

Acknowledgments. This research was supported by the Jet Propulsion Laboratory under contract 49-681-02081-0-8260. I appreciate conversations with P. MacDoran, K. Ong, H. Fliegel, J. McMillan, and J. Savage and a preprint kindly supplied by $P$. Bender. 1 thank $N$. Goulty and $D$. Anderson for a critical review of the manuscript. Contribution $\mathbf{2 6 2 5}$ of the Division of Geological and Planetary Sciences, California Institute of Technology, Pasadena, California.

\section{REFERENCES}

Anderson, D. L., and J. H. Whitcomb, The dilatancy-diffusion model of earthquake prediction, in Proceedings of the Conference on Tectonic Problems of the San Andreas Fault System, p. 417, Stanford University, Stanford, Calif., 1973.
Anderson, D. L., and J. H. Whitcomb, Time-dependent seismology, J. Geophys. Res., 80, 1497, 1975.

Bender, P. L., Applications of PTTI to new techniques for determining crustal movements, polar motion, and the rotation of the earth, paper presented at 6th Annual Precise Time and Time Interval Planning Meeting, Dep. of Def. and Nat. Aeronaut. and Space Admin., Washington, D. C., Dec. 3-5, 1974.

Castle, R. O., J. N. Alt, J. C. Savage, and E. I. Balazs, Elevation changes preceding the San Fernando earthquake of February 9, 1971, Geology, 2, 61, 1974.

Heiskanen, W. A., and H. Moritz, Physical Geodesy, 364 pp., W. H. Freeman, San Francisco, Calif., 1967.

Kennedy, G. C., and W. T. Holser, Pressure-volume-temperature and phase relations of water and carbon dioxide, in, Handbook of Physical Constants, Mem. 97, edited by S. P. Clark, Jr., p. 371, Geological Society of America, New York, 1966.

Kisslinger, C., Processes during the Matsushiro, Japan, earthquake swarm as revealed by levelling, gravity, and spring-flow observations, Geology. 3. 57, 1975.

MacDoran, P. F., Radio interferometry for international study of the earthquake mechanism, Acta Astronaut., L, 1427, 1974.

Nur, A., Dilatancy, pore fluids, and premonitory variations of $t_{g} / t_{p}$ travel times, Bull. Seismol. Soc. Amer., 62, 1217, 1972.

Nur, A., The Matsushiro earthquake swarm: A confirmation of the dilatancy-fluid flow model, Geology. 2, 217, 1974.

Ohtake, M., Changes in the ratio of compressional to shear wave velocity related with the occurrence of some shallow earthquakes in Japan, J. Phys. Earth, 2I, 173, 1973.

Oliver, H. W., L. C. Pakiser, ańd M. F. Kane, Gravity anomalies in the central Sierra Nevada, California, J. Geophys. Res., 66, 4265, 1961.

Oliver, H. W., S. L. Robbins, R. B. Grannell, R. W. Alewine, and S. Biehler, Surface and subsurface movements determined by remeasuring gravity, in San Fernando Earthquake-Geology and Geophysics, Bull. 196, p. 195, California Division of Mines and Geology, Sacramento, 1975.

Ong, K. M., P. F. MacDoran, J. B. Thomas, H. F. Fliegel, L. J. Skjerve, D. J. Spitzmesser, P. D. Batelaan, S. R. Paine, and M. G. Newsted, A demonstration of radio interferometric surveying using DSS 14 and the project Aries transportable antenna, JPL Deep Space Network Progr. Rep. 12-26, p. 41, Jet Propul, Lab., Pasadena, Calif., 1975a.

Ong, K. M., P. F. MacDoran, J. B. Thomas, H. F. Fliegel, L. J. Skjerve, D. J. Spitzmesser, P. D. Batelaan, S. R. Paine, and M. G. Newsted, A demonstration of a transportable radio interferometric system with $3-\mathrm{cm}$ accuracy on a $307-\mathrm{m}$ base line, submitted to $J$. Geophys. Res., 19756.

Ramsey, A. S., An Introduction to the Theory of Newtonian Attraction, 184 pp.. Cambridge University Press, New York, 1959.

Scholz, C. H., L. R. Sykes, and Y. P. Aggarwal, Earthquake prediction: A physical basis, Science, 181, 803, 1973.

Stuart, W. D., and M. J. S. Johnston, Intrusive origin of the Matsushiro earthquake swarm, Geology, 3, 63, 1975.

Tsubokawa, I., Y. Ogawa, and T. Hayashi, Crustal movements before and after the Niigata earthquake, J. Geod. Soc. Jap., 19, 165, 1964.

Whitcomb, J. H., J. D. Garmany, and D. L. Anderson, Earthquake prediction: Variation of seismic velocities before the San Fernando earthquake, Science, 180, 632, 1973.

(Received May 12, 1975; revised December 29, 1975; accepted March 29, 1976.) 